\title{
Assessing the transmission performance of iGMAS real-time data streams
}

\author{
Zhanke $\mathrm{He}^{1, \mathrm{a}}$, Wenda $\mathrm{Tang}^{2}$, Xuhai Yang ${ }^{1}$ and Liming Wang ${ }^{2}$ \\ ${ }^{1}$ National Time Service Center, Chinese Academy of Sciences, Xi'an, Shaanxi 710600, China \\ ${ }^{2}$ Xidian University, Xi'an, Shaanxi 710071, China
}

\begin{abstract}
The use of the BeiDou Navigation Satellite System (BDS) has been increasing in the Asian-Pacific region. Thus, the construction of the international GNSS Monitoring and Assessment System (iGMAS) is accelerating to meet the demanding requirements for highprecision real-time products. Latency and packet loss, two critical data transmission parameters, have great impact on the applications of real-time data streams. This paper aims to propose a Homology-Reverse method to investigate the data transmission performance of the iGMAS stations in local and wide area networks. The proposed method enables not only assessment on receivers but pretesting on network conditions of alternative tracking stations. This paper is expected to provide a reference for the construction of the iGMAS, Continuous Operational Reference System (CORS), and Satellite Based Augmentation Systems (SBAS).
\end{abstract}

\section{Introduction}

The rapid development of internet and Global Navigation Satellite System (GNSS) facilitates the widely use of real-time data streams of GNSS are flourishing in various fields, such as navigation and positioning, surveying and mapping, monitoring earthquake, meteorology, transportation, etc. The BeiDou Navigation Satellite System (BDS) indigenously launched by China officially started to serve the Asian-Pacific region since the late 2012. It is now being applied in a wide range of areas and markets with real-time data streams and related products. The international GNSS Monitoring and Assessment System (iGMAS) aims to establish a world-wide GNSS signal tracking network. It is designed to monitor the service performance and signal quality of multi-GNSS by high-precision receivers and high-gain omni-directional antenna. It provides services to customers worldwide [1]. At present, the iGMAS is boosting its pace of construction.

Data latency and packet loss, two critical parameters for transmission, play a key role in the utilization efficiency of real-time data streams. An analysis center always attempts to control the latency as small as possible to provide a more accurate feedback such as clock biases. Many international research institutions in the GNSS field are now exploring the real-time GNSS services with high accuracy. For example, the Jet Propulsion Laboratory (JPL) is the pioneer in this field [2]. The JPL products are transmitted to users with latency within 5 seconds [3-5]. Wuhan University conducted real-time data analysis using PANDA $[6,7]$. In their prototype system, the latency of the data streams transmitted from the observation stations to the data center were controlled within 2-5

\footnotetext{
${ }^{\mathrm{a}}$ Corresponding author : hzk@ntsc.ac.cn
} 
seconds after synchronization [3]. The transmission performance of real-time data flows is essential for iGMAS, Continuous Operational Reference System (CORS), and the Satellite Based Augmentation Systems (SBAS), which motivates further study in this paper.

The latency of real-time data streams is composed of network transmission delays, software and hardware processing delays. The former depends on the transmission distance, network type and bandwidth. The hardware processing delays are primarily sourced from the signal processing and output of receivers, while the software processing delay is caused by the data processing and analysis delays of the NTRIP Server, Caster and Client [8]. The packet loss is defined as the ratio of the packets discarded to the total number of the packets sent, or the ratio of the number of packets in the RINEX form to the total number of packets theoretically received. The daily packet number (86400s) was used in this paper.

"Ping method" is commonly used to measure the network latency and packet loss ratio. This involves sending one ping per second between hosts and counting how many times the request is lost. Unfortunately, the "Ping method" for analysing loss is prone to inaccurate measurements due to its statistical limitations. Even in small amounts, packet loss will reduce application throughput, degrade or halt streaming applications, and introduce lags for interactive applications. The Ping method is based on the Internet Control Message Protocol (ICMP) whereas the NTRIP is based on the Transmission Control Protocol (TCP) that includes a connection-oriented service, a reliable data transfer service and a congestion-control mechanism [9]. In addition, the real-time transmission latency and packet loss are different from Ping results because of its various and complicated delay sources. Currently, signal receivers of the four iGMAS systems (BDS, GPS, GLONASS and Galileo) have being improved. Tracking stations are at the stage of site selection and construction and the network types are being chosen. There are notable differences in latency of real-time data output by receivers. The latency will occur during no matter the data transmission from tracking stations to data centers satisfies the requirements or not. Therefore, it is of great importance to study the assessment method of latency and packet loss generated in real-time data streams.

The huge latency of data transmission generated in individual stations may lead to network congestion and thus has a great impact on the use of real-time data streams. This paper proposes a Homology-Reverse method to measure and analyse the transmission latency from domestic tracking stations (see Table 1) to data centers using optimized software [10]. The proposed method is to provide a reference for using the BeiDou real-time data streams.

Table 1. Approximate coordinates of iGMAS domestic tracking stations.

\begin{tabular}{|c|c|c|c|}
\hline Site & Latitude & Longitude & Altitude (m) \\
\hline XIA1 & $\mathrm{N} 34.369^{\circ}$ & E109.221 ${ }^{\circ}$ & 449.46 \\
\hline CHU1 & $\mathrm{N} 43.791^{\circ}$ & E125.443 ${ }^{\circ}$ & 273.67 \\
\hline SHA1 & $\mathrm{N} 31.099^{\circ}$ & E121.200 & 21.74 \\
\hline GUA1 & $\mathrm{N} 43.471^{\circ}$ & $\mathrm{E} 87.177^{\circ}$ & 2028.82 \\
\hline BJF1 & $\mathrm{N} 39.607^{\circ}$ & E115.892 & 75.52 \\
\hline WUH1 & $\mathrm{N} 30.515^{\circ}$ & E114.490 & 71.24 \\
\hline LHA1 & $\mathrm{N} 29.657^{\circ}$ & E91.104 & 3625.10 \\
\hline KUN1 & $\mathrm{N} 25.030^{\circ}$ & E102.797 & 1988.08 \\
\hline
\end{tabular}

\section{Methods and materials}

A general assessment strategy is to make a comparison of tested receivers to internationally recognized reference receivers. Real-time data streams are sent out by the NTRIP Server of receivers, retransmitted by the NTRIP Caster in data centers, and decoded by the NTRIP Client in data centers or analysis centers. The data latency and packet loss are then measured. Time synchronization must be 
maintained between receivers and data center servers. The latency of real-time signals output by receivers can be evaluated in the local area network (LAN). For Wide Area Network (WAN), network conditions between tracking stations and data centers (e.g. network congestion) can be examined by fixing the receivers in tracking stations. Nevertheless, the examination in WAN requires two or more receivers. Thus, its cost is high and the efficiency is low.

The so-called Homology-Reverse method is to establish a recognized receiver and data center in LAN. Real-time data streams sent out from the NTRIP Server of the receiver are retransferred by NTRIP Caster in data centers. After data decoding by the NTRIP Client installed in the receiver or computer, the latency and packet loss are then measured. Time synchronization must be guaranteed among receivers, servers and computers in data center. This proposed method can not only evaluate the performance of receivers being improved but pretest network of tracking stations. Unlike the conventional method in WAN, the proposed method has advantages of low cost and high efficiency.

The "backward" and "forward" latency are the same since the same round-trip paths are taken. For assessing the round-trip latency, a Homology-Returned method can be used by simply exchanging the position of the NTRIP Caster and the NTRIP Client used in the Homology-Reverse method.

As shown in Figure 1, the assessment system consists of GNSS stations, reference stations, data centers, and analysis centers. GNSS stations can be further classified into test stations and base stations. A base station, consisting of receivers, antennae, etc., is able to provide measured data and navigation ephemeris encoded and transmitted in RTCM real-time signals by the NTRIP Server. A test station has receivers or computers on which the NTRIP Client is running. Data centers and analysis centers include data receiving server, data processing server, and time synchronization server. The NTRIP Casters are installed in the data receiving server. Time synchronization server, traced back to UTC (NTSC) provides the time synchronization service for tracking stations and data centers.

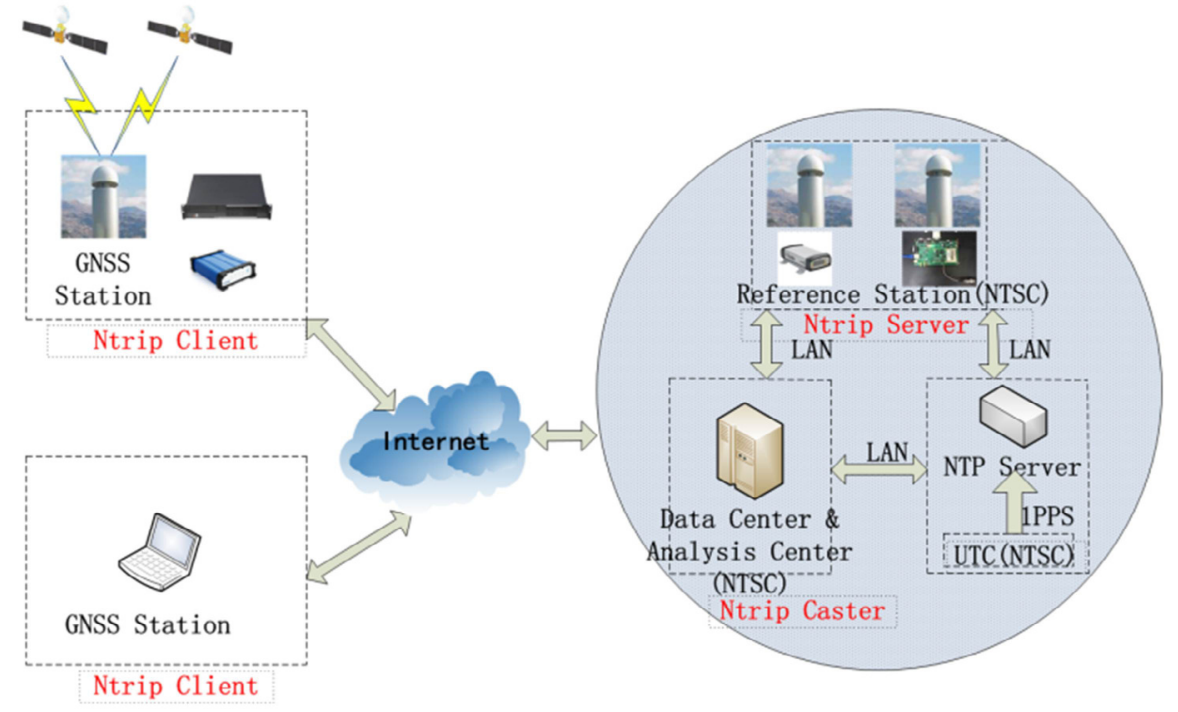

Figure 1. Schematic of the assessment system.

For precise point positioning, the acquisition of update information such as clock biases is affected by many factors [11] (see Figure 2). It involves signal processing and output delays of receivers in tracking stations, encoding delays of NTRIP Server, network transmission delays from stations to data centers, retransmission delays of NTRIP Caster in data centers, decoding delays of NTRIP Client, data analysis delays, delays of update information transmitted from analysis centers to clients, user positioning calculation delays, etc. This study only focuses on the real-time data latency in the data center, including the first five data latency mentioned above. 

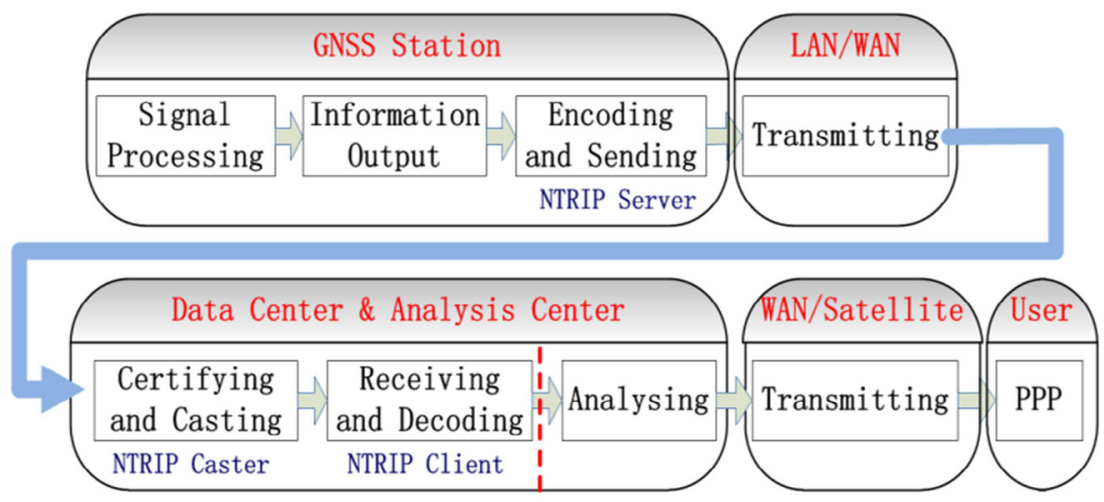

Figure 2. Main sources of Latency.

\section{Results and discussion}

\subsection{Reference receivers}

Trimble NetR9 was used as a Reference receiver. The NTRIP Server of receivers sent out RTCM realtime data retransferred by the NTRIP Caster of data centers. Latency was measured after data decoding by the NTRIP Client. Figure 3 shows the measured latency over 24 hours. Table 2 lists the statistical results from the Ping method and the NTRIP software. The real-time data streams output by Reference receivers have a latency of $28-182 \mathrm{~ms}$, with an average value of $38.99 \mathrm{~ms}$. By adding timestamp on the data packets, the time consumptions of LAN transmission, NTRIP Caster transfer and NTRIP Client decoding were measured, respectively. All the measurements were less than $1 \mathrm{~ms}$. Therefore, the latency of real-time data streams mainly caused by the receiver output latency and network transmission conditions.
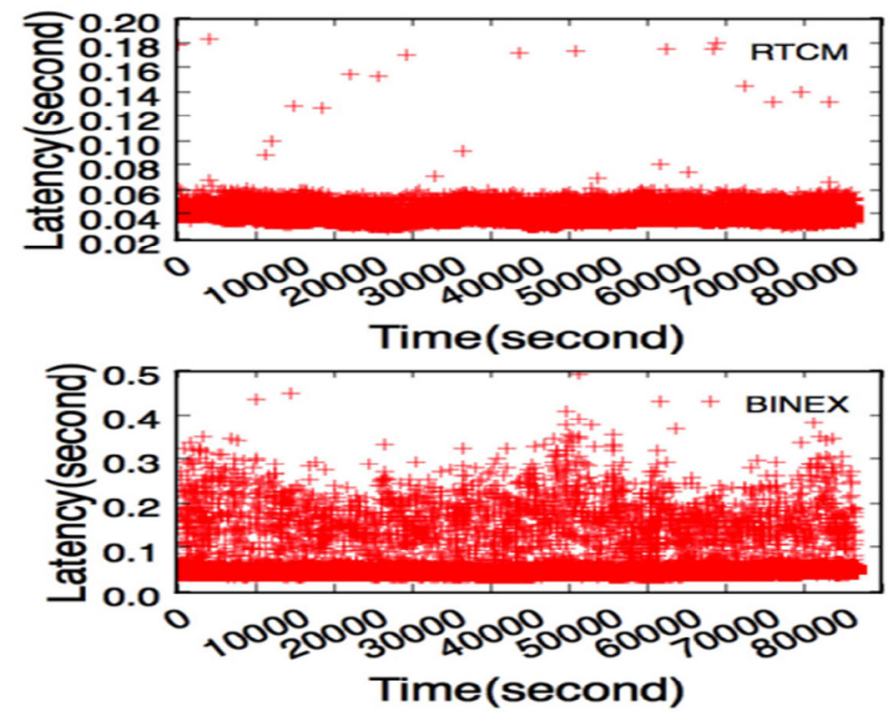

Figure 3. Latency of RTCM and BINEX real-time data streams in LAN. 
Table 2. Comparison of latency and packet loss from Ping and NTRIP software in LAN.

\begin{tabular}{|c|c|c|c|c|}
\hline Types & $\begin{array}{c}\text { Min } \\
\text { Latency } \\
\text { (ms) }\end{array}$ & $\begin{array}{c}\text { Max } \\
\text { Latency } \\
(\mathbf{m s})\end{array}$ & $\begin{array}{c}\text { Avg } \\
\text { Latency } \\
\text { (ms) }\end{array}$ & $\begin{array}{c}\text { Packet } \\
\text { loss(s) }\end{array}$ \\
\hline $\begin{array}{c}\text { Ping(round-trip } \\
\text { delay time) }\end{array}$ & 0.21 & 1.355 & 0.274 & 0 \\
\hline $\begin{array}{c}\text { NTRIP Software } \\
\text { (RTCM) }\end{array}$ & 28 & 182 & 38.99 & 0 \\
\hline $\begin{array}{c}\text { NTRIP Software } \\
\text { (BINEX) }\end{array}$ & 29 & 491 & 46.53 & 0 \\
\hline
\end{tabular}

On the other hand, BINEX real-time data streams sent out by the NTRIP Caster of Trimble NetR9 were received and decoded by the NTRIP Client [12], transcoded by the BINEX2RTCM, encoded by the NTRIP Server, and finally sent out by the RTCM. The bottom of Figure 3 shows the latency of BINEX. It can be seen that the BINEX latency is much larger than the RTCM latency. In addition, for a receiver with four systems, under the current constellation distribution for each navigation system, the BINEX data quantity is $1.19 \mathrm{kB}$ per second whereas RTCM data is $0.47 \mathrm{kB}$ per second. The total differences for one day can reach $60.75 \mathrm{MB}$. The application of the RTCM real-time data streams greatly reduces the Network transmission pressure.

\subsection{Test stations}

Generally, the latency and packet loss measured based on the proposed assessment method are consistent with the practical measurements. However, the practically measured latency is a slightly larger than that from the proposed method. The XIA1 station used UB370 (OEM plate), selfdeveloped NTRIP Server, Data center and analysis center in LAN. The data latency of the tested receiver was in basic agreement with the Reference receiver, as shown in the top of Figure 4 (A represents assessment results). For the BJF1 station, the measured mean latency of data streams is $1 \mathrm{~s}$ greater than the assessed results. This means the tested receiver has relatively large data latency. Therefore, the proposed assessment method can be used to determine the source of data latency. If the assessed latency was the same as the measured results, the latency is expected to be from a network that should be checked to satisfy the station requirements. If not as shown in the bottom of Figure 4, the latency should come from the receiver.

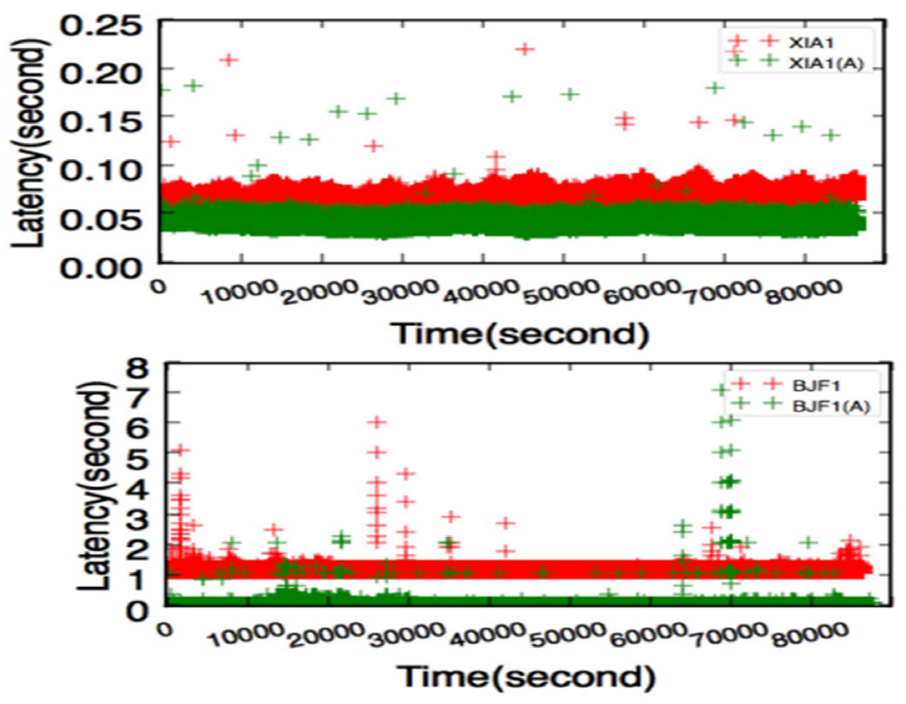

Figure 4. Comparison of measured and evaluated real-time data latency in the XIA1 and BJF1stations. 


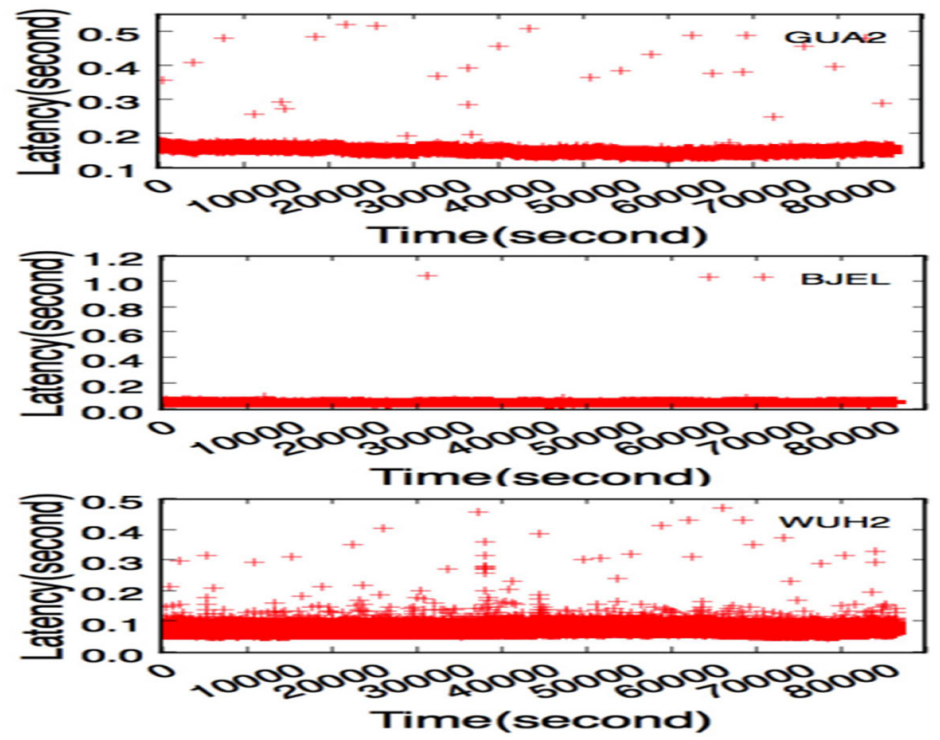

Figure 5. Real-time data latency in the GUA2, BJEL and WUH2 (SDH) stations.

Table 3. Latency and packet loss from Ping in WAN.

\begin{tabular}{|c|c|c|c|c|}
\hline Sites & $\begin{array}{c}\text { Min } \\
\text { Latency } \\
(\mathbf{m s})\end{array}$ & $\begin{array}{c}\text { Max } \\
\text { Latency } \\
(\mathbf{m s})\end{array}$ & $\begin{array}{c}\text { Avg } \\
\text { Latency } \\
(\mathbf{m s})\end{array}$ & $\begin{array}{c}\text { Packet } \\
\text { loss(s) }\end{array}$ \\
\hline XIA1 & 0.17 & 11.96 & 0.365 & 33 \\
\hline GUA2 & 65.54 & 135.25 & 66.71 & 41 \\
\hline BJEL & 13.27 & 127.59 & 16.74 & 4 \\
\hline BJF1 & 19.4 & 361.57 & 45.86 & 235 \\
\hline WUH2 & 24.78 & 266.16 & 31.34 & 1 \\
\hline
\end{tabular}

Table 4. Latency and packet loss from NTRIP software in WAN.

\begin{tabular}{|c|c|c|c|c|}
\hline Sites & $\begin{array}{c}\text { Min } \\
\text { Latency } \\
(\mathbf{m s})\end{array}$ & $\begin{array}{c}\text { Max } \\
\text { Latency } \\
(\mathbf{m s})\end{array}$ & $\begin{array}{c}\text { Avg } \\
\text { Latency } \\
(\mathbf{m s})\end{array}$ & $\begin{array}{c}\text { Packet } \\
\text { loss(s) }\end{array}$ \\
\hline XIA1 & 43 & 218 & 67.74 & 0 \\
\hline GUA2 & 124 & 519 & 150.2 & 0 \\
\hline BJEL & 35 & 1041 & 45.9 & 0 \\
\hline BJF1 & 1027 & 5988 & 1072 & 0 \\
\hline WUH2 & 45 & 467 & 65.75 & 0 \\
\hline
\end{tabular}

Synchronous Digital Hierarchy (SDH) Network is safe, reliable and has low latency but costly. System builders should think twice before choosing SDH Network (MSTP) or Virtual Private Network (VPN). The WUH2 station used the SDH Network (MSTP) to connect data center and analysis center. Its latency is illustrated in Figure 5. In addition, both BJEL and GUA2 stations from 
China Science and Technology Network (CSTNET) were used. Their latency and packet loss agree well with special lines. Table 3 and 4 list the results from Ping and NTRIP software. Network conditions play a key role in the latency and packet losses. Several common internets have the same amount of latency and packet loss as the special line. This is against the fact that common networks have larger latency and packet loss than special lines.

\section{3 iGMAS stations}

The data center and analysis center deploys the China Science and Technology Network. Assessments were conducted at eight iGMAS tracking stations (XIA1, BJF1, SHA1, KUN1, SHA1, WUH1, GUA1 and LHA1) and two overseas tracking stations (CNYR and ZHON). The measurement work lasted one week and each day had similar results. Figure 6 10 show the measured results one sample day. Each tracking station provides broadband at a speed no less than $2 \mathrm{Mbps}$. The domestic network operators include the China Science and Technology Network, the China Unicom Network (CUNET) and the China Telecom Network (CTNET). The latency and packet losses measured from Ping and NTRIP software are shown in Table 5 and 6.
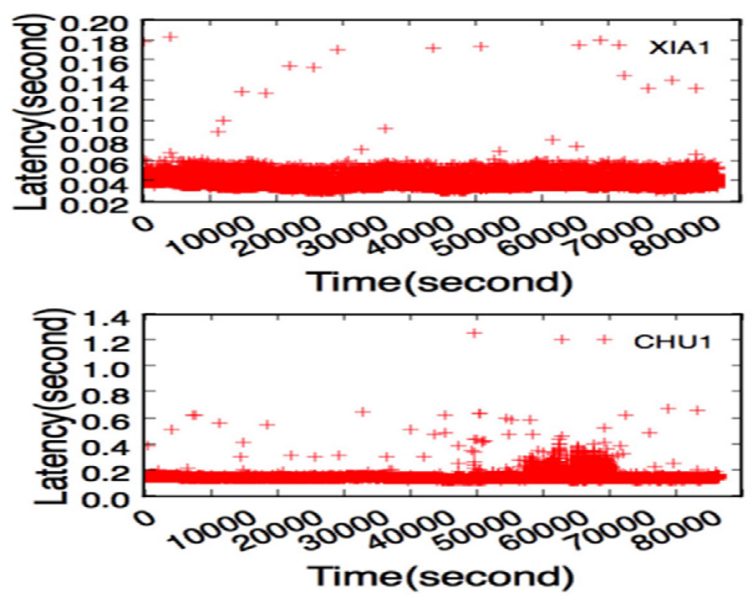

Figure 6. Real-time data latency in the XIA1 and CHU1 stations.
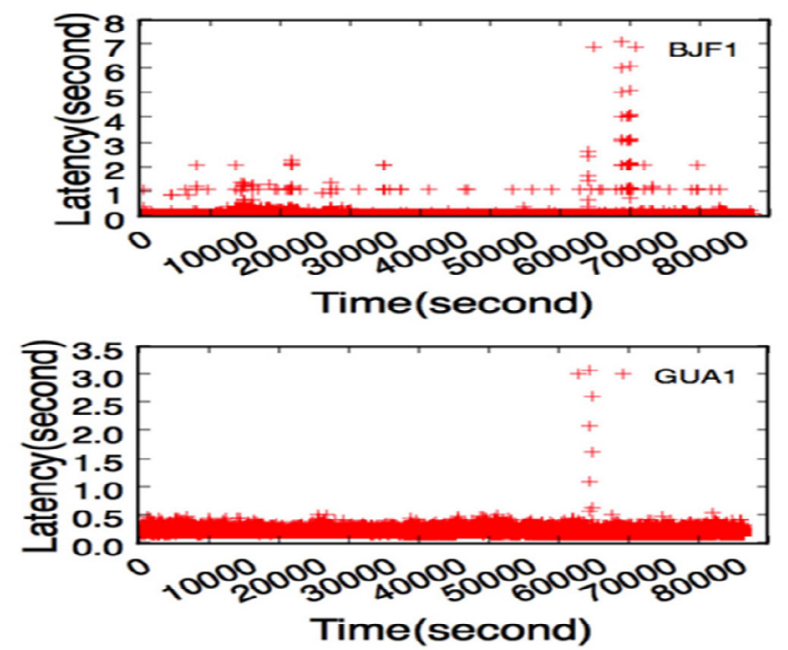

Figure 7. Real-time data latency in the BJF1 and GUA1 stations. 

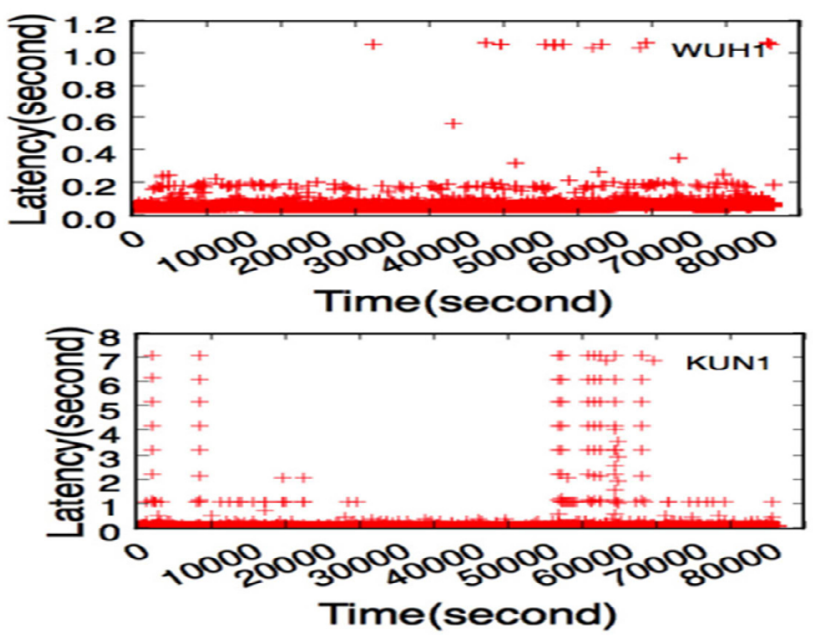

Figure 8. Real-time data latency in the WUH1 and KUN1 stations.

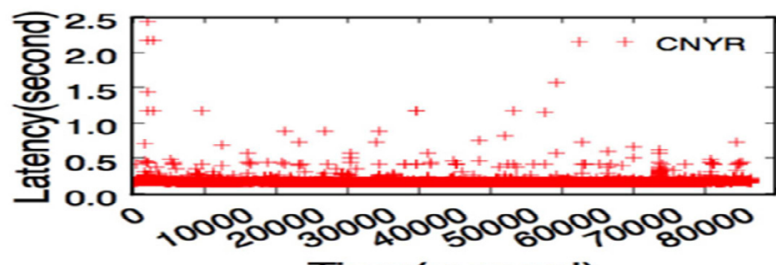

Time(second)

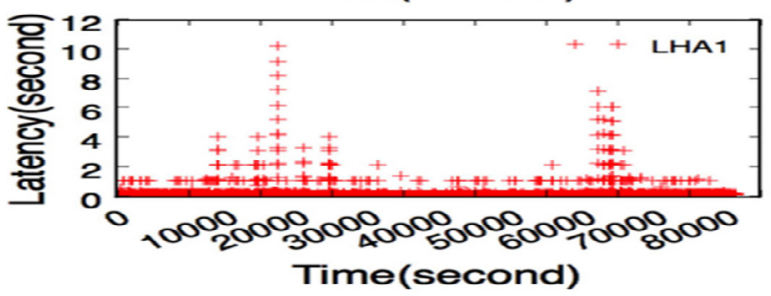

Figure 9. Real-time data latency in the CNYR and LHA1 stations.
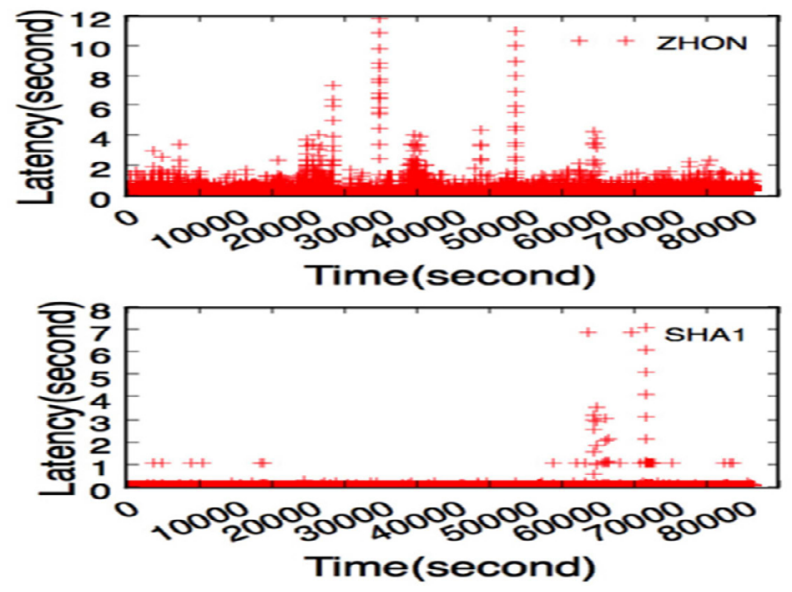

Figure 10. Real-time data latency in the ZHON and SHA1 stations. 
No packet loss occurs in all the domestic stations except KUN1 and LHA1. Despite network congestion at some stations, $99.62 \%$ of the latency is controlled within 1 s and $99.82 \%$ within 2 s. The overseas station ZHON has no packet loss whereas CNYR does. However, more than $99.40 \%$ of the latency in the two stations was controlled within 1s. It is observed that some stations have network congestion and latency as high as 10 s. The reason should be further studied.

The short-term instable characteristics of clock biases bring a certain error after a short-term extrapolation. The extrapolation duration should be reduced as much as possible. Therefore, the waiting time for estimating the clock biases should be short enough considering timeliness. However, to ensure a sufficient amount of measurements for each assessment, reasonable estimation waiting time must be set to maintain the stability and high precision of the estimation and prediction of clock biases. The majority of the data from the iGMAS domestic network arrives at the data center and analysis center within 1 seconds of its BeiDou time-tag. Data with latency less than 1 second are used in computing the BeiDou clocks.

Table 5. Latency and packet loss from ping of tested iGMAS stations.

\begin{tabular}{|c|c|c|c|c|}
\hline Sites & $\begin{array}{c}\text { Min } \\
\text { Latency } \\
(\mathbf{m s})\end{array}$ & $\begin{array}{c}\text { Max } \\
\text { Latency } \\
(\mathbf{m s})\end{array}$ & $\begin{array}{c}\text { Avg } \\
\text { Latency } \\
(\mathbf{m s})\end{array}$ & $\begin{array}{c}\text { Packet } \\
\text { loss(s) }\end{array}$ \\
\hline XIA1(A) & 0.17 & 11.96 & 0.365 & 33 \\
\hline CHU1(A) & 32.81 & 698.2 & 41.26 & 55 \\
\hline SHA1(A) & 37.85 & 63.06 & 42.23 & 19 \\
\hline GUA1(A) & 66.84 & 132.68 & 67.97 & 43 \\
\hline BJF1(A) & 19.4 & 361.57 & 45.86 & 235 \\
\hline WUH1(A) & 38.03 & 175.09 & 40.94 & 157 \\
\hline LHA1(A) & 69.56 & 381.86 & 75.14 & 23 \\
\hline KUN1(A) & 69.55 & 843.59 & 71.06 & 230 \\
\hline ZHON(A) & 42.68 & 73.95 & 43.49 & 32 \\
\hline CNYR(A) & 233.9 & 341.03 & 245.2 & 23 \\
\hline
\end{tabular}

Table 6. Latency and packet loss from NTRIP software tested iGMAS stations.

\begin{tabular}{|c|c|c|c|c|}
\hline Sites & $\begin{array}{c}\text { Min } \\
\text { Latency } \\
(\mathbf{m s})\end{array}$ & $\begin{array}{c}\text { Max } \\
\text { Latency } \\
(\mathbf{m s})\end{array}$ & $\begin{array}{c}\text { Avg } \\
\text { Latncy } \\
(\mathbf{m s})\end{array}$ & $\begin{array}{c}\text { Packet } \\
\text { loss(s) }\end{array}$ \\
\hline XIA1(A) & 43 & 218 & 67.74 & 0 \\
\hline CHU1(A) & 111 & 1254 & 138.4 & 0 \\
\hline SHA1(A) & 48 & 7067 & 62.58 & 0 \\
\hline GUA1(A) & 125 & 3065 & 154.2 & 0 \\
\hline BJF1(A) & 45 & 3996 & 66.77 & 0 \\
\hline WUH1(A) & 41 & 3054 & 53.04 & 0 \\
\hline LHA1(A) & 65 & 10180 & 85.93 & 84 \\
\hline KUN1(A) & 66 & 7080 & 82.84 & 41 \\
\hline ZHON(A) & 313 & 11792 & 362.7 & 0 \\
\hline CNYR(A) & 156 & 2437 & 170 & 173 \\
\hline
\end{tabular}

\section{Conclusion}

According to the measured and evaluated results, the RTCM transmission of real-time data streams significantly reduces the network transmission pressure. Some stations have the same amount of 
latency and packet loss by using either internet or special lines. For the current internet conditions, packet loss can be eliminated for real-time data streams. The latency of $99.4 \%$ of data packets is controlled within 1s. The use of internet can satisfy the requirements of the iGMAS, CORS, and SBAS for improving data transmission performance. In the NTRIP software, the encryption scheme enables the enhancement of safety and privacy as well as optimization of operation costs. The findings in this paper offer a reference for the use of the BeiDou real-time data streams as well as the facility construction.

\section{Acknowledgements}

This work was supported by the National Natural Science Foundation (11173026), the international GNSS Monitoring and Assessment System (iGMAS), the West Light Foundation of the Chinese Academy of Sciences (2013YB10), and the Fundamental Research Funds for the Central Universities.

\section{References}

1. W.H. Jiao, Q. Ding, J.W. Li, X.C. Lu, L.P. Feng, J.Q. Ma, and G. Chen, Monitoring and assessment of GNSS open services, Journal of Navigation, 64, S19-S29 (2011)

2. R.J. Muellerschoen, W.I. Bertiger, and M.F. Lough, Results of an internet-based dual-frequency global differential GPS system, Proceedings of the Iain World Congress \& Annual Meeting of the Institute of Navigation, 796-802 (San Diego, USA, 2000)

3. C. Shi, Y.D. Lou, W.W. Song, S.F. Gu, C.J. Geng, W.T. Yi, and Y.Y. Liu, A wide area real-time differential GPS prototype system in China and result analysis, Survey Review, 43 (322), 351360 (2011)

4. R.J. Muellerschoen, A. Reichert, D. Kuang, M. Heflin, W. Bertiger, and Y. Bar-Sever, Orbit determination with NASA's high accuracy real-time global differential GPS system, Proceedings of the 14th International Technical Meeting of the Satellite Division of the Institute of Navigation (ION GPS 2001), 2294-2303 (Salt Lake City, USA, 2001)

5. M.O. Kechine, C.C.J.M. Tiberius, and H.V.D. Marel, An experimental performance analysis of real-time kinematic positioning with NASA's Internet-Based Global Differential GPS, GPS solutions, 8 (1), 9-22 (2004)

6. J.N. Liu and M.R. Ge, PANDA software and its preliminary result of positioning and orbit determination, Wuhan University Journal of Natural Sciences, 8 (2), 603-609 (2003)

7. C. Shi, Q.L. Zhao, J.H. Geng, Y.D. Lou, M.R. Ge, and J.N. Liu, Recent development of PANDA software in GNSS data processing, Proc. SPIE. International Conference on Earth Observation Data Processing and Analysis (ICEODPA) (eds. D.R. Li, J.Y. Gong, and H.Y Wu), 7285, 72851S1-1 (Wuhan, China, 2008)

8. G. Weber, D. Dettmering, H. Gebhard, and R. Kalafus, Networked transport of RTCM via internet protocol (Ntrip) ... IP-Streaming for Real-Time GNSS Applications, Proceedings of the 18th International Technical Meeting of the Satellite Division of the Institute of Navigation (ION GNSS 2005), 2243-2247 (Long Beach, USA, 2005)

9. J.F. Kurose and K.W. Ross, Computer Networking. 6th edition, Pearson Education (New Jersey, USA, 2012)

10. Z.K. He, W.D. Tang, X.H. Yang, L.M. Wang, and J.H. Liu, Use of NTRIP for optimizing the decoding algorithm for real-time data streams, Sensors, 14 (10), 18878-18885 (2014)

11. M. Li, L.Z. Qu, Q.L. Zhao, J. Guo, X. Su, and X.T. Li, Precise point positioning with the BeiDou navigation satellite system, Sensors, 14 (1), 927-943 (2014)

12. R. Galas and W. Kohler, A binary exchange format for GPS data, Physics and Chemistry of the Earth Part A Solid Earth and Geodesy, 26, 645-648 (2001) 